\title{
Zener Double Exchange from Local Valence Fluctuations in Magnetite
}

\author{
R. J. McQueeney, ${ }^{1,2}$ M. Yethiraj, ${ }^{3, *}$ S. Chang, ${ }^{2}$ W. Montfrooij, ${ }^{4}$ T. G. Perring, ${ }^{5,7}$ J. M. Honig, ${ }^{6}$ and P. Metcalf ${ }^{6}$ \\ ${ }^{1}$ Department of Physics \& Astronomy, Iowa State University, Ames, Iowa 50011 USA \\ ${ }^{2}$ Ames Laboratory, Ames, Iowa 50011 USA \\ ${ }^{3}$ Center for Neutron Scattering, Oak Ridge National Laboratory, Oak Ridge, Tennessee 37831 USA \\ ${ }^{4}$ Department of Physics and Missouri Research Reactor, University of Missouri, Columbia, Missouri 65211 USA \\ ${ }^{5}$ ISIS Facility, Rutherford Appleton Laboratory, Didcot, Oxfordshire, OX11 OQX, United Kingdom \\ ${ }^{6}$ Department of Chemistry, Purdue University, West Lafayette, Indiana 47907 USA \\ ${ }^{7}$ Department of Physics and Astronomy, University College London, Gower Street, London WC1E6BT, United Kingdom
}

(Received 19 July 2007; published 10 December 2007; publisher error corrected 5 February 2008)

\begin{abstract}
Magnetite $\left(\mathrm{Fe}_{3} \mathrm{O}_{4}\right)$ is a mixed valent system where electronic conductivity occurs on the $B$ site (octahedral) iron sublattice of the spinel structure. Below $T_{V}=123 \mathrm{~K}$, a metal-insulator transition occurs which is argued to arise from the charge ordering of $2+$ and $3+$ iron valences on the $B$ sites (Verwey transition). Inelastic neutron scattering measurements show that optical spin waves propagating on the $B$ site sublattice $(\sim 80 \mathrm{meV})$ are shifted upwards in energy above $T_{V}$ due to the occurrence of $B$ - $B$ ferromagnetic double exchange in the mixed valent phase. The double exchange interaction affects only spin waves of $\Delta_{5}$ symmetry, not all modes, indicating that valence fluctuations are slow and the double exchange is constrained by short-range electron correlations above $T_{V}$.
\end{abstract}

DOI: 10.1103/PhysRevLett.99.246401

Magnetite $\left(\mathrm{Fe}_{3} \mathrm{O}_{4}\right)$ is the prototypical example of a metal-insulator transition with a charge-ordered (CO) insulating ground state (Verwey transition). Since its discovery nearly 70 years ago [1], the driving forces behind the Verwey transition are still not completely understood $[2,3]$. Magnetite has a cubic inverse spinel crystal structure containing two different symmetry iron sites: the $A$ site resides in tetrahedrally coordinated oxygen interstices and has stable valence $\left(3 d^{5}, \mathrm{Fe}^{3+}\right)$; the two $B$ sites have octahedral coordination and a fractional average valence of $2.5+$. The ferrimagnetic structure consists of ferromagnetic $A$ - and $B$-sublattices aligned antiparallel to each other $\left(T_{C}=858 \mathrm{~K}\right)$. Below $T_{V}=123 \mathrm{~K}$, magnetite undergoes a metal-insulator transition resulting in a decrease of the conductivity by two orders-of-magnitude. The model that has persisted over time is that extra electrons forming $\mathrm{Fe}^{2+}$ ions $\left(3 d^{6}\right)$ hop to neighboring $\mathrm{Fe}^{3+}$ sites on the cornershared $B$-sublattice tetrahedral network and give rise to electrical conductivity. Anderson argued that short-range ordering of $\mathrm{Fe}^{2+}$ and $\mathrm{Fe}^{3+}$ exists above $T_{V}$ due to significant intersite Coulomb repulsion and frustration on the $B$ site sublattice [4]. The short-ranged electron correlations maintain local charge "neutrality" $(2.5+$ average valence on each tetrahedron), thereby restricting charge hopping and conductivity [5]. In the classic picture of the Verwey transtion, Coulomb repulsions win out at low temperatures, resulting in long-range $\mathrm{CO}$ of $\mathrm{Fe}^{2+}$ and $\mathrm{Fe}^{3+}$ [6] in a process reminiscent of Wigner crystallization [7]. However, elastic and orbital interactions [8] induce monoclinic lattice distortions whose complexity has made characterization of the Verwey state difficult [9]. Even the validity of the CO model has been questioned recently [10], but neutron [11] and resonant $\mathrm{x}$-ray scattering measurements [12] now appear to converge on fractional CO.
PACS numbers: 71.30.+h, 75.30.Ds, 75.30.Et, 78.70.Nx

In this Letter, we provide strong evidence for Anderson's original picture of short-ranged electron correlations in the mixed valent (MV) phase. Valence fluctuations occurring on the $B$-sublattice modify the magnetic exchange and affect spin waves propagating on $\mathrm{Fe} B$ sites. Inelastic neutron scattering measurements reveal that $B$ site optical spin waves are shifted up in energy and broadened above $T_{V}$ due to ferromagnetic double exchange (DE). Ferromagnetic DE arises from real charge transfer processes in MV materials, a good example being the ferromagnetic metallic state in the manganites [13]. For fast electron hopping in the band limit, cubic symmetry demands that the average DE should uniformly affect all $B-B$ pairs. However, our results show that only spin waves of a particular symmetry are affected by DE, implying the presence of electron hopping restricted by short-ranged charge correlations. The observed correlations give indirect evidence that intersite Coulomb interactions play a role in the Verwey transition.

Inelastic neutron scattering measurements were performed on the MAPS instrument at the ISIS facility at Rutherford Appleton Laboratory on a single-crystal of $\mathrm{Fe}_{3} \mathrm{O}_{4}$ weighing $\sim 10$ grams $\left(T_{V}=123 \mathrm{~K}\right)$. Details of sample preparation and characterization are given elsewhere [16]. The sample was mounted with cubic [HK0] as the primary scattering plane, and data were collected with an incident neutron energy of $160 \mathrm{meV}$ and the incident beam at an angle of $25^{\circ}$ from the [110] axis. Time-of-flight neutron spectra were collected at $110 \mathrm{~K}$ $\left(T<T_{V}\right)$ and $130 \mathrm{~K}\left(T>T_{V}\right)$ and scattered intensities were histogrammed into energy transfer $(\hbar \omega)$ and momentum transfer $(\hbar \mathbf{Q})$ bins. Data were subsequently analyzed using MSLICE [17] and TOBYFIT [18] computer programs. 


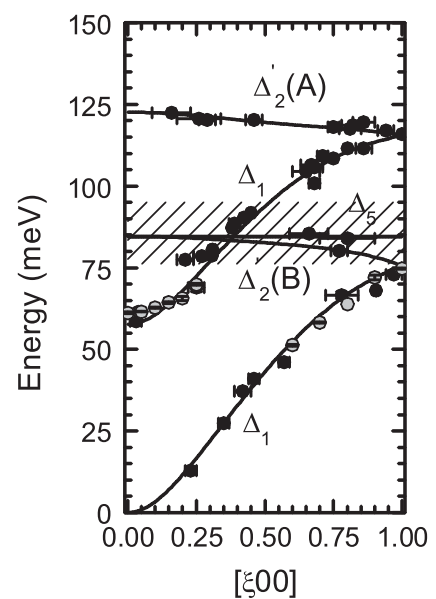

FIG. 1. Spin wave dispersion of magnetite above $T_{V}$. Black (gray) symbols are inelastic neutron scattering data from MAPS (HB-3). Lines are results from a Heisenberg model with parameters discussed in the main text. The hatched area contains very broad $B$ site spin waves.

The measured neutron scattering spectra from MAPS (and also the HB-3 spectrometer at the High Flux Isotope Reactor at Oak Ridge National Laboratory) were used to determine the spin wave dispersion in magnetite. In the cubic spinel phase above $T_{V}$, the primitive rhombohedral unit cell contains six iron atoms $(2 A$ and $4 B)$, leading to six spin wave branches. Figure 1 shows the spin wave dispersion along [100] as determined from Gaussian fits to spin wave modes [19]. The dispersion is well represented by a Heisenberg model where antiferromagnetic $A B$ superexchange (SE), via oxygen, is dominant $\left(J_{A B}=\right.$ $-4.8 \mathrm{meV}$ ) and is responsible for ferrimagnetism. Ferromagnetic BB exchange arises from a combination of SE, $\mathrm{DE}$, and direct exchange and is much weaker $\left(J_{B B}=\right.$
$0.69 \mathrm{meV})$. Weak antiferromagnetic nearest- and nextnearest-neighbor $A A$ exchange is also present $\left(J_{A A}^{(1)}=\right.$ $\left.-0.35 \mathrm{meV}, J_{A A}^{(2)}=-0.2 \mathrm{meV}\right)$. The dispersion calculated from a Heisenberg model with exchange parameters above and spins $S_{A}=2.5$ and $S_{B}=2.25$ is also shown in Fig. 1 . Branch symmetries were identified from the model spin wave eigenvectors and are given the following labels and descriptions: $\Delta_{1}$ (acoustic and steeply dispersing optic), $\Delta_{2}^{\prime}(A)$ (optic spin wave on $A$-sublattice), $\Delta_{2}^{\prime}(B)$ (optic spin wave on $B$-sublattice), and $\Delta_{5}$ (doubly-degenerate optic spin waves on $B$-sublattice). Modes of $\Delta_{5}$ and $\Delta_{2}^{\prime}(B)$ symmetry at $\sim 80 \mathrm{meV}$ (in the hatched region of Fig. 1) propagate solely on the $B$-sublattice and were observed to be very broad and weak, as discussed below.

Figures 2(a) and 2(b) show images of scattered intensity in the $[H K 0]$ plane at 130 and $110 \mathrm{~K}$, respectively. The arcs and rings in the images correspond to the intersection of the neutron measurement surface and the dispersion surfaces. Figure 2(c) shows a calculation of the MAPS data using the Heisenberg model described above. Calculations simulate the sampling of spin waves in $(\mathbf{Q}, \omega)$ space performed by MAPS. Features of the calculated and measured spectra are in good agreement (as anticipated from Fig. 1) with one notable exception. As indicated in Fig. 2(a), the measured $B$ site optical spin wave branch at $\sim 80 \mathrm{meV}$ is poorly defined in the region between [220] and [420] at $130 \mathrm{~K}$. Comparison with the $110 \mathrm{~K}$ spectrum illustrates two features: (1) the $B$ site modes sharpen up below $T_{V}$ in better agreement with the cubic model (despite the lowering of crystallographic symmetry), and (2) other spin wave branches are essentially unaffected, indicating that $J_{A B}$ and $J_{A A}$ are insensitive to the Verwey transition.

To study the $B$ site modes more closely, energy cuts were made through the MAPS data at different values of $K$ and (a) Data, $\mathrm{T}=130 \mathrm{~K}$

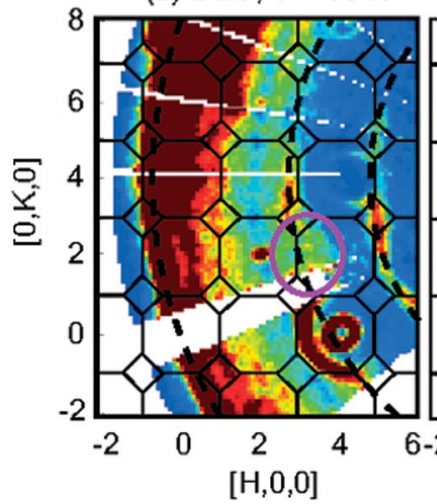

(b) Data, $\mathrm{T}=110 \mathrm{~K}$

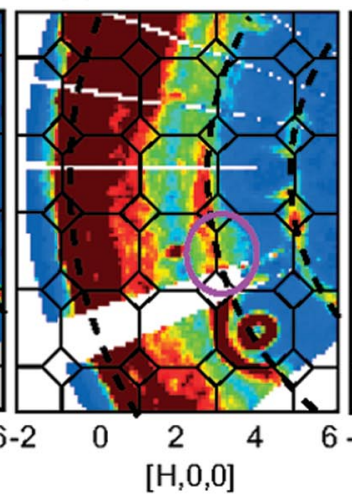

(c) Calculation

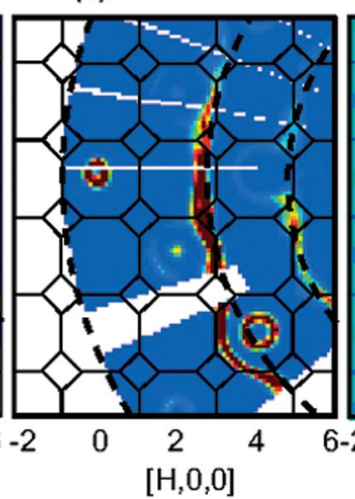

(d) Symmetry

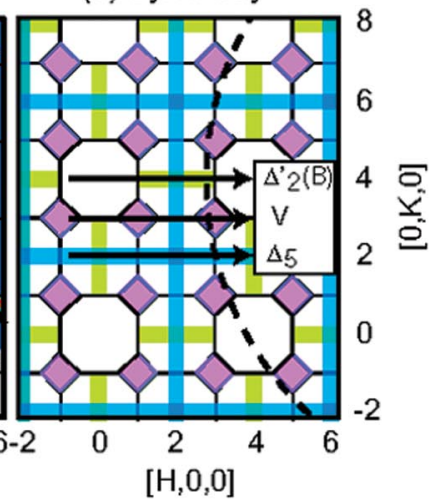

FIG. 2 (color). (a) Neutron scattering intensity in the [HKO] plane at $130 \mathrm{~K}$. The color scale indicates neutron intensity (red the highest). Thin lines show the Brillouin zone boundaries of the cubic phase. Curved, dashed lines show 0,80 , and 120 meV energy transfers (from left to right). The purple circled region indicates extremely broad and weak $\Delta_{5}$ symmetry optical spin waves at 80 meV. (b) The $110 \mathrm{~K}$ data set below $T_{V}$. In the purple circled region, the $\Delta_{5}$ mode is more pronounced. (c) Calculation of the MAPS spin wave spectra using a Heisenberg model with $J_{B B}=0.44 \mathrm{meV}(T=110 \mathrm{~K})$. Data and calculations have been smoothed by a boxcar averaging procedure. (d) Symmetry of $B$ site optical spin waves in different Brillouin zones at $80 \mathrm{meV} ; \Delta_{5}$ (blue), $\Delta_{2}^{\prime}(B)$ (green), $V$ (purple). Energy cuts shown in Fig. 3 are indicated by arrows. 


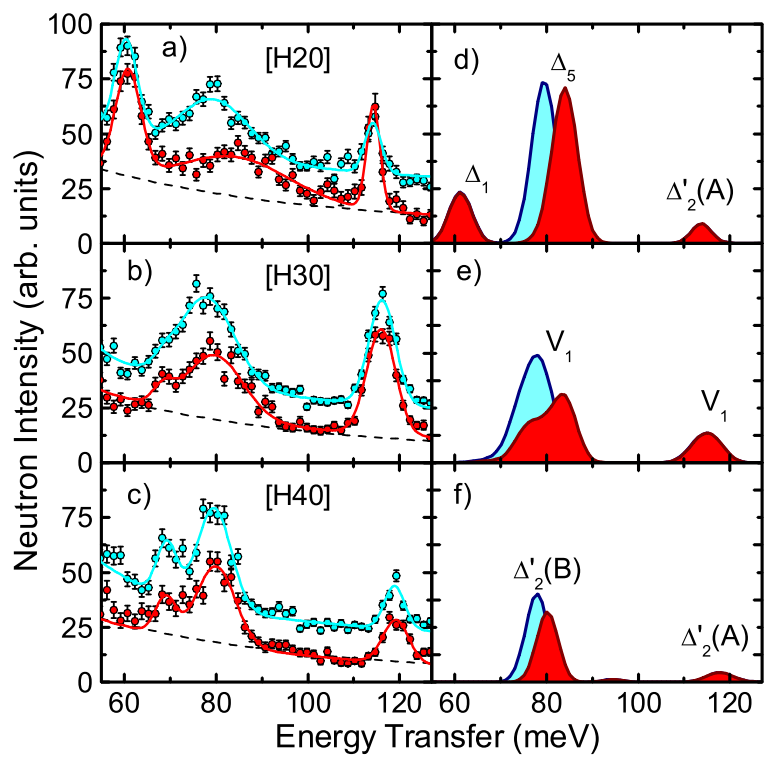

FIG. 3 (color online). Inelastic neutron scattering intensity as a function of energy transfer for cuts along (a) [H20], (b) [H30], and (c) [H40]. Upper (lower) symbols indicate measurements done at $T=110 \mathrm{~K}(130 \mathrm{~K})$. Lines are Gaussian fits to the respective data. The dashed line is the background estimated from cuts along [H60]. The $110 \mathrm{~K}$ plots are offset vertically for clarity. Panels (d)-(f) show the identical cuts as (a)-(c) as calculated from a Heisenberg model for magnetite with $J_{B B}=$ $0.44 \mathrm{meV}$ (light, blue online) and $J_{B B}=0.69 \mathrm{meV}$ (dark, red online). Spin wave modes are labeled by symmetry.

different temperatures, as shown in Fig. 3. To improve statistics, cuts are obtained by averaging neutron intensity over a range of reciprocal space. Figure 3 shows the following cuts made in the range $-0.25 \leq L \leq 0.25$ : [H20] (1.75 $\leq K \leq 2.25)$, [H30] $(2.75 \leq K \leq 3.25)$, and [H40] $(3.75 \leq K \leq 4.25)$. These reciprocal space cuts allow for specific symmetry selection of the $B$ site optical spin waves, as shown in Fig. 2(d). In the constrained $(\mathbf{Q}, \hbar \omega)$ measurement surface of MAPS, $H$ is a function of $\hbar \omega$, as can be ascertained from the comparison of Figs. 2 and 3. The energy resolution full-width-at-half-maximum (FWHM) is calculated to be $3-4 \mathrm{meV}$ for dispersionless modes in the energy range shown (using the TOBYFIT program). In all cuts, spin wave modes at 60 and $117 \mathrm{meV}$ are independent of temperature and resolution limited, while $\sim 80 \mathrm{meV}$ modes are broader than the resolution. The energy cut along the [H20] direction in Fig. 3(a) shows that the $\Delta_{5}$ symmetry branch at $\sim 80 \mathrm{meV}$ shifts up in energy and becomes severely broadened above $T_{V}$, as also apparent in Fig. 2. Gaussian fits to the data indicate that the mode energy shifts from 79.6(5) to 84(1) $\mathrm{meV}$ and the FWHM broadens by $60 \%$ from 17 to $28 \mathrm{meV}$ above $T_{V}$. The $B$ site spin waves along [H30] in Fig. 3(b) ( $V$ symmetry [20]) and [H40] in Fig. 3(c) $\left(\Delta_{2}^{\prime}(B)\right.$ symmetry) also have broad line shapes with FWHM of 14 and $10 \mathrm{meV}$, respectively, but relatively little temperature dependence. We also note the weak peak near $70 \mathrm{meV}$ in Fig. 3(c) is likely to be a phonon, based on examination of higher- $\mathbf{Q}$ data.

The anomalous $B$ site spin waves can be explained by consideration of ferromagnetic DE. The conduction electron associated with the $\mathrm{Fe}^{2+}$ ion is forced to remain oppositely aligned to the $\mathrm{Fe}^{3+} 3 d^{5}$ core spin by the Pauli exclusion principle (effectively $J_{\text {Hund }} \rightarrow \infty$ ). Since the $B$-sublattice is already ferromagnetically aligned due to strong $J_{A B}$, spin polarized conduction results in DE [21] and leads to an increase in $J_{B B}$. In turn, the energy of $B$ site optical spin waves increases. The enhancement of DE above $T_{V}$ can be estimated from the energy of the dispersionless $\Delta_{5}$ mode, $6 J_{A B} S_{B}+8 J_{B B} S_{B}$. Since $J_{A B}$ does not change [16], the shift of $4.4 \mathrm{meV}$ implies that $J_{B B}$ increases from 0.44 to $0.69 \mathrm{meV}$. The energy cuts are compared to the Heisenberg model structure factor calculation in Figs. 3(d)-3(f) with $J_{B B}=0.44 \mathrm{meV} \quad\left(T<T_{V}\right)$ and $0.69 \mathrm{meV}\left(T>T_{V}\right)$. Comparison to calculation shows that (1) all $B$ site spin wave peaks are much weaker and broader than Heisenberg model predictions, and (2) changing $J_{B B}$ in the model shifts the energy of all mode symmetries, in disagreement with the observation that only the $\Delta_{5}$ mode has a large temperature dependence. Structure factors in the energy range $75 \leq \hbar \omega \leq 90 \mathrm{meV}$ and $115 \leq$ $\hbar \omega \leq 120 \mathrm{meV}$ and $L$-range $-0.25 \leq L \leq 0.25$ are shown in Fig. 4 as a function of $K$. Figure 4(a) further illustrates the anomalous $\Delta_{5}$ mode, which shows a minimum in the measured structure factor whereas the calculation predicts a maximum. Figure 4(b) indicates that $A$ site optical spin waves have little temperature dependence and agree quite well with Heisenberg calculations. The disagreement between data and model structure factor calculations for the $B$ site modes reflects a failure of the Heisenberg model for only the broadest (most strongly coupled) modes.

It is clear that stiffening and extreme broadening of only the $\Delta_{5}$ modes above $T_{V}$ cannot be explained by uniformly enhanced BB coupling as might be expected from a band
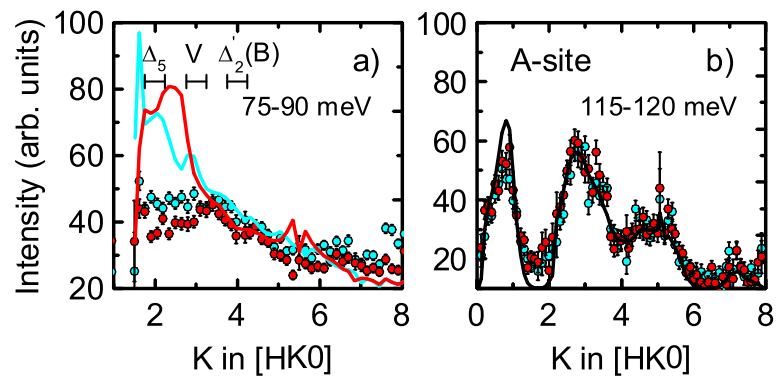

FIG. 4 (color online). (a) Neutron structure factor for $B$ site optical spin waves averaged from $75-90 \mathrm{meV}$. Data are shown at $130 \mathrm{~K}$ (dark circles, red online) and $110 \mathrm{~K}$ (light circles, blue online). Heisenberg model calculations are shown with $J_{B B}=$ $0.69 \mathrm{meV}$ (dark line, red online) and $0.44 \mathrm{meV}$ (light line, blue online). Horizontal bars indicate dominant symmetry. (b) Structure factor for $A$ site optical spin waves from 115-120 meV. Line is calculated from a Heisenberg model. 

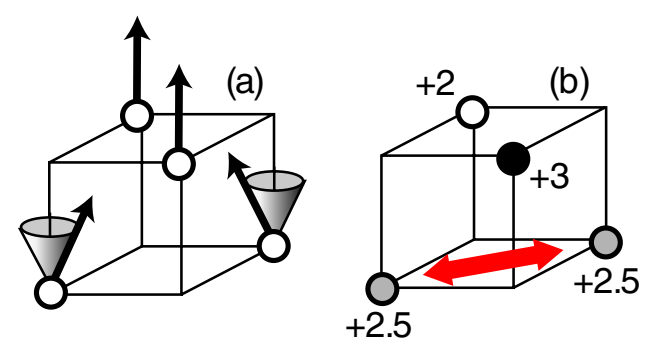

FIG. 5 (color online). Schematic drawing of (a) spin and (b) charge modes on the $B$-sublattice with $\Delta_{5}$ symmetry. In (b), the heavy arrow indicates direction of charge fluctuations.

approach. The failure of a band model is reiterated by estimating the DE arising from metallic conductivity, $J_{\mathrm{DE}} \sim n t / 2 \sqrt{2} S_{B}^{2}[22,23]$ where $n \approx 1 / 2$ is the concentration of charge carriers in the conduction band and $t$ the electron hopping integral. The effective one-band hopping integral from local spin density approximation (LSDA) calculation is $\sim 130 \mathrm{meV}$ [24], leading to an estimate of $J_{\mathrm{DE}} \sim 5 \mathrm{meV}$ that is significantly larger than the observed value $\left(J_{\mathrm{DE}} \approx J_{B B}\left(T>T_{V}\right)-J_{B B}\left(T<T_{V}\right)=0.25 \mathrm{meV}\right)$. This disagreement emphasizes the importance of charge correlation and/or polaronic effects above $T_{V}$ that lead to activated conduction and a reduction of the effective hopping integral. This picture is supported by optical conductivity measurements showing that the $\sim 100 \mathrm{meV}$ electronic energy gap below $T_{V}$ (which suppresses DE) is replaced by a pseudogap above $T_{V}[25,26]$. In this limit, the Zener DE picture of slow and local charge hopping between distinct valence states above $T_{V}$ is appropriate, and the closing of the electronic gap allows the coupling of spin waves to valence fluctuations leading to broadened spin wave line shapes. We also conclude that no features in the spin wave spectrum below $T_{V}$ can be definitively associated with long-range $\mathrm{CO}$, in agreement with the results of our previous study [16].

The predominant $\Delta_{5}$ spin wave symmetry implies that valence fluctuations have the same symmetry and occur along the quasi-one-dimensional [110] $B$ site chains, as illustrated in Fig. 5. The spin wave changes the alignment of neighboring spins, thereby impeding valence fluctuations which favor parallel spins. The presence of slow $\Delta_{5}$ valence fluctuations coupling to the lattice has also been observed with neutron diffuse scattering and occur well above $T_{V}$ [27]. In the MV phase, these local symmetrybreaking charge correlations are short-ranged. While we interpret our data based on the symmetry assignments for modes along the [100] direction, broadening everywhere in the Brillouin zone indicates that the coupled $B$ site charge and spin fluctuations are local. The resulting picture of valence fluctuations constrained by short-range ordering of Fe valences is similar to that proposed originally by Anderson [4]. In addition, the implication of slow electron hopping $(\$ 80 \mathrm{meV})$ supports more detailed theories of magnetite that invoke polaronic behavior for the $\mathrm{Fe}^{2+}$ charge carriers [5]. Spin-charge coupling is large and may contribute to the polaronic binding energy. Finally, although the spin waves are insensitive to the details of long-range $\mathrm{CO}$ below $T_{V}$, the $\Delta_{5}$ symmetry of charge correlations above $T_{V}$ is similar to the order parameter of the Verwey state, which has been argued to be a combination of $\Delta_{5} \oplus X_{3}$ cubic representations [15]. This indirectly supports the idea that intersite Coulomb interactions play a role in the Verwey transition.

R. J. M. would like to thank S. Satpathy for useful discussions. Work is supported by the U. S. Department of Energy Office of Science under the following contracts: Ames Laboratory under Contract No. DE-AC0207CH11358, Oak Ridge National Laboratory, which is managed by UT-Batelle LLC, under Contract No. DEAC00OR22725.

*Current address: Bragg Institute, ANSTO, Lucas Heights, NSW 2234 Australia

[1] E. J. W. Verwey, Nature (London) 144, 327 (1939).

[2] F. Walz, J. Phys. Condens. Matter 14, R285 (2002).

[3] J. García and G. Subías, J. Phys. Condens. Matter 16, R145 (2004)

[4] P. W. Anderson, Phys. Rev. 102, 1008 (1956).

[5] D. Ihle and B. Lorenz, J. Phys. C 19, 5239 (1986).

[6] E. J. Verwey, P. W. Haayman, and F. C. Romeijn, J. Chem. Phys. 15, 181 (1947).

[7] N. F. Mott, Adv. Phys. 16, 49 (1967).

[8] I. Leonov et al., Phys. Rev. Lett. 93, 146404 (2004).

[9] J. M. Zuo, J. C. H. Spence, and W. Petuskey, Phys. Rev. B 42, 8451 (1990).

[10] J. García et al., Phys. Rev. Lett. 85, 578 (2000).

[11] J. P. Wright, J. P. Attfield, and P. G. Radaelli, Phys. Rev. B 66, 214422 (2002).

[12] E. Nazarenko et al., Phys. Rev. Lett. 97, 056403 (2006).

[13] Y. Tokura, Rep. Prog. Phys. 69, 797 (2006).

[14] A. Rosencwaig, Phys. Rev. 181, 946 (1969).

[15] P. Piekarz, K. Parlinski, and A. M. Oles, Phys. Rev. Lett. 97, 156402 (2006).

[16] R. J. McQueeney et al., Phys. Rev. B 73, 174409 (2006).

[17] R. Coldea, computer code MSLICE: A data analysis programme for time-of-flight neutron spectrometers, 2004.

[18] T. G. Perring, computer code TOBYFIT-Least-Squares Fitting to Single Crystal Data on HET, MARI, and MAPS, 2004.

[19] Use of Gaussian fits, rather than resolution convolved fits, may lead to small errors in the fitted energy for steeply dispersing modes.

[20] $V$ symmetry direction is [1 $\xi 0]$, on the Brillouin zone face.

[21] J. Loos and P. Novák, Phys. Rev. B 66, 132403 (2002).

[22] A. J. Millis, P. B. Littlewood, and B. I. Shraiman, Phys. Rev. Lett. 74, 5144 (1995).

[23] N. Furukawa, J. Phys. Soc. Jpn. 65, 1174 (1996).

[24] Z. Zhang and S. Satpathy, Phys. Rev. B 44, 13319 (1991).

[25] L. Degiorgi, P. Wachter, and D. Ihle, Phys. Rev. B 35, 9259 (1987).

[26] S. K. Park, T. Ishikawa, and Y. Tokura, Phys. Rev. B 58, 3717 (1998).

[27] S. M. Shapiro, M. Iizumi, and G. Shirane, Phys. Rev. B 14, 200 (1976). 